\title{
A Long Right Atrial Thrombus Intermittently Prolapsing into Right Ventricle in a Patient with Recurrent Ischemic Stroke: An Image Report
}

\author{
Zhigang Liu1, Salman M. Malik',2, James S. Gammie³, Boaz D. Rosen1,2* \\ ${ }^{1}$ Department of Medicine, MedStar Harbor Hospital, Baltimore, USA \\ ${ }^{2}$ Division of Cardiology, MedStar Harbor Hospital, Baltimore, USA \\ ${ }^{3}$ Division of Cardiac Surgery, University of Maryland Medical Center, Baltimore, USA \\ Email: rosen.boaz@gmail.com
}

Received 5 April 2014; revised 1 May 2014; accepted 18 May 2014

Copyright (C) 2014 by authors and Scientific Research Publishing Inc.

This work is licensed under the Creative Commons Attribution International License (CC BY). http://creativecommons.org/licenses/by/4.0/

(c) (i) Open Access

\begin{abstract}
A 39-year-old male was admitted for recurrent ischemic strokes. TEE was performed to detect the potential intra-cardiac thrombi and right-to-left shunts. A large hypermobile, echodense, irregular mass was found in the right atrium, which looked like a cluster of grapes. The motion of the components of the mass was synchronized, prolapsing into right ventricle in an octopus-like fashion during diastole. There was no evidence for PFO or other intra-cardiac shunts by color flow Doppler. The patient was referred to surgery and a continuous $30 \mathrm{~cm}$ long thrombus spanning from the inferior vena cava to the right ventricle was discovered.
\end{abstract}

Keywords

Right Atrial Thrombus, Ischemic Stroke, Thrombus in Transit

\section{Introduction}

The right atiral masses are most likely to be either tumor or thrombi [1]. Among the tumors in the right atrium, metastatic tumors, from breast, lung, lymphoma, melanoma or sarcomas, are 20 times more common than primary tumors [2]. Although myxomas are classically seen in the left atrium, it is not unusual for them to be detected in the right atrium.

Three types of right atrial thrombi are described. Type A thrombi are highly mobile with a serpiginous mor-

${ }^{*}$ Corresponding author.

How to cite this paper: Liu, Z., et al. (2014) A Long Right Atrial Thrombus Intermittently Prolapsing into Right Ventricle in a Patient with Recurrent Ischemic Stroke: An Image Report. Case Reports in Clinical Medicine, 3, 309-313.

http://dx.doi.org/10.4236/crcm.2014.35068 
phology. They are associated with deep venous thrombosis and pulmonary embolism, and are thought to derive from large veins. Type B thrombi origin from the right atrium due to some cardiac abnormalities, and thus are non-mobile. Type $\mathrm{C}$ thrombi are rare with morphology similar to myxoma, but they are highly mobile.

The differentiation between the right atrial tumor and thrombi is difficult and often impossible without invasive interventions, despite the wide availability of echocardiography, CT scan and magnetic resonance imaging [3] [4].

In this case, we report a Type A right atrial thrombus confirmed by pathology examination. The morphology of this thrombus is unusual with a synchronized octopus-like prolapsing into the right ventricle during diastole.

\section{Case Presentation}

A 39-year-old African-American male was admitted for sudden onset of left-sided weakness with the upper extremity being weaker than the lower extremity. He had a past medical history significant for hypertension diagnosed 25 years ago, and non-ischemic cardiomyopathy with ejection fraction 15\% diagnosed with cardiac catheterization two months ago. Home medication included hydralazine, amlodipine, lisinopril, metoprolol, spironolactone, and furosemide. No significant family history exists except that mother had hypertension. No smoking, alcohol or illicit drug abuse.

In ED patient's vital signs were as following: temperature 37.1, respiration rate 22, pulse 110, blood pressure 184/127, and oxygen saturation 98\% on room air. Positive physical examination included: lungs, decreased breath sound on the base; heart, a grade 2/6 systolic murmur at the apex; on neurological examination, moderate dysarthria, marked left facial droop, partial deviation of the tongue to the left, left hand strength 0/5, proximal left upper extremity strength $3 / 5$, hypoactive deep tendon reflexes throughout. Initial lab results included: PT 14.8, INR 1.2, PTT 32.2, BNP 263, troponin 0.023, CK MB 0.7, CK 178, CBC within normal limits, BMP within normal limits, total cholesterol 174, HDL 32, LDL 121, triglyceride 105, urine toxicology screening negative. ECG revealed sinus tachycardia with occasional isolated PVCs, left ventricular hypertrophy, nonspecific ST-T changes related to LVH.

An initial CT head without contrast revealed an acute infarction in the right frontal lobe measuring $6 \times 3 \mathrm{~cm}$. A transthoracic echocardiography (TTE) was performed to rule out the cardiogenic ischemic stroke, and showed markedly dilated left ventricle with severe left ventricular systolic dysfunction. However, no obvious intra-cardiac thrombus was seen. Carotid duplex scan was negative for significant stenosis or plaque. Follow-up brain MRI and MRA without contrast two days later showed an evolving large right frontal lobe acute infarction and a second new infarction in the left superior frontal lobe, extending up to the right corona radiata. A transesophageal echocardiography (TEE) was performed for the high suspicions of cardiac source of emboli. In addition to the findings noticed by TTE, TEE demonstrated left ventricle and atrium spontaneous echo contrast without obvious thrombi in the left atrium or ventricle. There was hypertrabeculation of the LV wall suggestive of LV noncompaction as the etiology of his non-ischemic cardiomyopathy (Figure 1, movie clip 1). A large hyper-mobile,

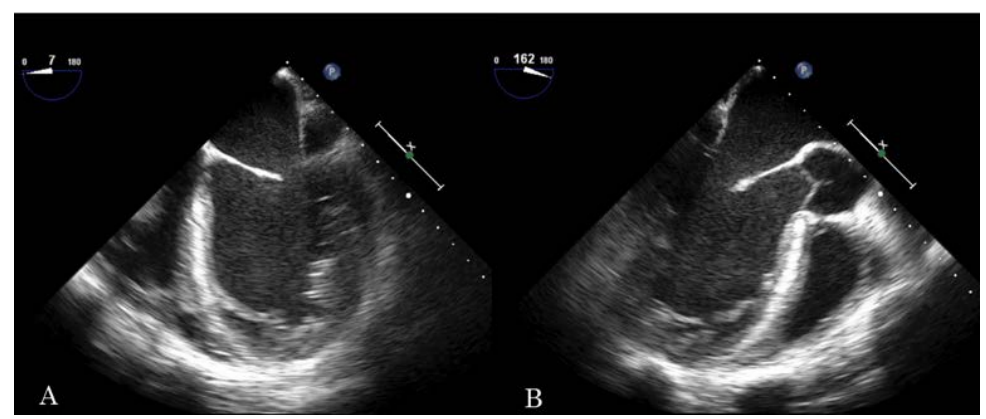

Figure 1. A: mid-esophageal four chamber view. Left ventricle is enlarged with severe global LV dysfunction with global hypokinesis. Hypertrabeculation of the LV wall, suggestive of non-compaction as a cause of the cardiomyopathy. Left atrium is enlarged. Spontaneous contrast in the LV and left atrium. No thrombus is seen in these chambers. Right ventricular function is impaired as well. A hyperechogenic mass, likely thrombus, is seen in the right atrium. B: mid-esophageal long axis view. Note again a spontaneous contrast in the left ventricle. 
echodense, irregular mass was found in the right atrium, which looked like a cluster of grapes (Figure 2, movie clip 2). The point of attachment was difficult to detect, but it seemed to originate from the inferior vane caca. The motion of the components of the mass was synchronized, prolapsing into right ventricle in an octopus-like fashion during diastole (Figure 3, movie clip 3). No evidence of PFO was found by color flow Doppler. Bubble study was not performed due to the concern of dislodging the right atrium mass. No other intra-cardiac shunts, or significant atherosclerosis of the aorta were noticed either.

The patient was referred to surgery and a continuous $30 \mathrm{~cm}$ long thrombus spanning from the inferior vena cava to the right ventricle was discovered, and was successfully removed. No evidence of any right to left shunt was found during the surgery.

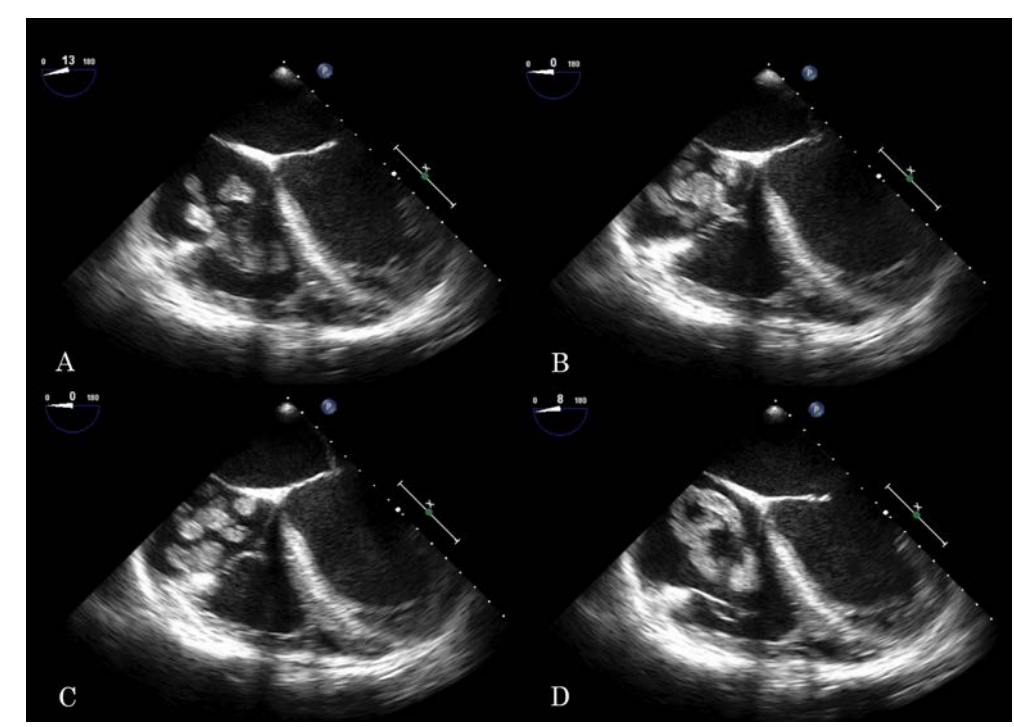

Figure 2. Four chamber mid-esophageal view with slight tilt of the probe to the right. Right atrium and ventricle are enlarged. RV function is impaired. A large hyperechogenic mass, likely thrombus, is seen in the right atrium, prolapsing to the right ventricle during diastole. Its morphology in this view is similar to a cluster of grapes. The synchronized motion of its components, suggests that this is a single elongated structure.

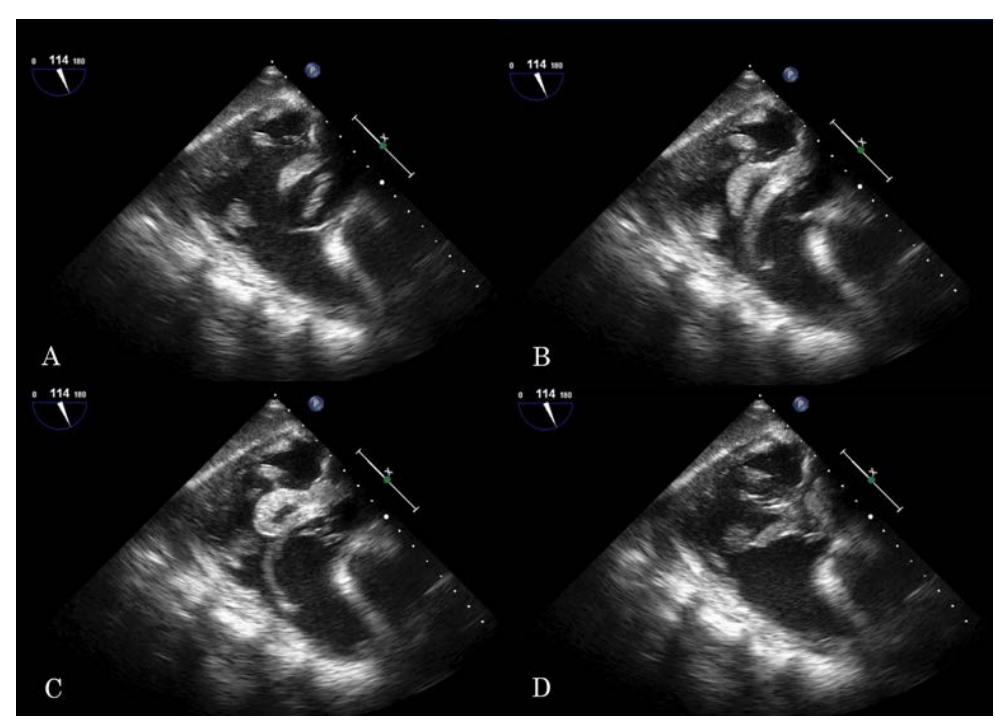

Figure 3. Transgastric right ventricular inflow-outflow view. Note the large hyperechogenic mass (i.e. thrombus) prolapsing deep into the right ventricle. This mass has an octopus-like morphology. 


\section{Discussion}

The patient had a Type A right atrial thrombus, with which the pulmonary thromboembolism rate ranging between 79\% (documented) and 98\% (clinical diagnosis) [5]. However, no pulmonary thromboembolism was detected by chest CT angiography. In addition, Doppler failed to detect any lower extremity deep venous thrombus. A real time 3D echocardiography might be a better module to evaluate this long right atrial thrombus compared to regular TEE [6] [7]. The transthoracic echocardiogram on admission did not detect any right atrial thrombus. All these findings indicated that the long right atrial thrombus was a newly formed, or may have been missed due to the limited quality of the transthoracic echo done in the ICU on a ventilated patient.

The recurrent ischemic strokes did not arise from the right atrial thrombus, with no evidence of any right to left shunt being noticed during the surgery. It was possible that some transient and unstable thrombi developed in the left heart or beyond and then were dislodged to the central nervous system leading to the recurrent ischemic strokes. In addition, the presence of spontaneous echo contrast in the left ventricle and atrium is also associated with higher risk of ischemic stroke [8].

Despite the fact that the patient's ischemic stroke did not arise from the right atrial thrombi, we need to keep in mind that paradoxical emboli are not uncommon causes of ischemic stroke in the presence of right-to-left shunts [9]. TEE may not able to detect all the right-to left shunts, given the relatively high false negative rate of TEE [10]. Transcranial Doppler sonography (TCD) may be performed to further detect any right-to-left shunt missed by TEE due to the smaller size [11]. Finally, there is a remote possibility of paradoxical embolism through an undetected pulmonary arterio-venous malformation, but this is unlikely.

No consensus has been made concerning how to treat right atrial thrombi. The algorithms we have now are based on analyses of isolated case reports or case series [12]. Prospective case series and registry data also provide some insights. For Type A and Type C, which are mobile, the thrombus usually resolves rapid after thrombolytic therapy with rt-PA [13]. In addition, the short time lag required to disperse the clot after thrombolytic therapy makes it imperative to delay any decision about new aggressive therapy [14]. Surgery was performed on our patient, however, for both diagnostic and therapeutic purposes. Moreover, the patient is not a candidate for thrombolytic therapy with recent recurrent ischemic strokes. When it comes to Type B thrombi, cautions are given to thrombolytic agents with the concerns that these agents may prompt distal embolism by dissolving the adherent stalk. If the Type B thrombi are giant, a few case reports suggest that an open surgical intervention is the preferred therapy [12] [15] [16].

\section{References}

[1] Lam, K.Y., Dickens, P. and Chan, A.C. (1993) Tumors of the Heart. A 20-Year Experience with a Review of 12,485 Consecutive Autopsies. Archives of Pathology \& Laboratory Medicine, 117, 1027-1031.

[2] Chitwood Jr., W.R. (1988) Cardiac Neoplasms: Current Diagnosis, Pathology, and Therapy. Journal of Cardiac Surgery, 3, 119-154. http://dx.doi.org/10.1111/j.1540-8191.1988.tb00232.x

[3] Aksu, T., et al. (2008) Thrombi in the Right Atrium and Inferior Vena Cava Mimicking Myxoma in a Patient with Recurrent Pulmonary Thromboembolism. Turk Kardiyoloji Dernegi Arsivi, 36, 555-557.

[4] Delange Segura, L. (2005) Limitations of Transesophageal Ultrasound in the Assessment of Intracardiac Masses: A Case Report. ta Española de Anestesiología y Reanimación, 52, 421-424.

[5] Farfel, Z., et al. (1987) Review of Echocardiographically Diagnosed Right Heart Entrapment of Pulmonary Emboli-inTransit with Emphasis on Management. American Heart Journal, 113, 171-178. http://dx.doi.org/10.1016/0002-8703(87)90026-3

[6] Betrian Blasco, P., et al. (2008) Three-Dimensional Imaging of Redundant Chiari’s Network Prolapsing into Right Ventricle. Journal of the American Society of Echocardiography, 21, 188e1-188e2.

[7] Wertman, B.M., et al. (2008) Right Atrial and Ventricular Masses of Unknown Origin. Journal of the American Society of Echocardiography, 21, 776e5-776e7.

[8] Siddiqui, M.A., Holmberg, M.J. and Khan, I.A. (2001) Spontaneous Echo Contrast in Left Atrial Appendage during Sinus Rhythm. Texas Heart Institute Journal, 28, 322-323.

[9] Harvey, J.R., et al. (1986) Clinically Silent Atrial Septal Defects with Evidence for Cerebral Embolization. Annals of Internal Medicine, 105, 695-697. http://dx.doi.org/10.7326/0003-4819-105-5-695

[10] Martinez-Sanchez, P., et al. (2012) Low Sensitivity of the Echocardiograph Compared with Contrast Transcranial Doppler in Right-to-Left Shunt]. Neurologia, 27, 61-67. http://dx.doi.org/10.1016/j.nrl.2011.05.010 
[11] Droste, D.W., et al. (2004) Right-to-Left-Shunts Detected by Transesophageal Echocardiography and Transcranial Doppler Sonography. Cerebrovasc Disease, 17, 191-196. http://dx.doi.org/10.1159/000075790

[12] Rose, P.S., Punjabi, N.M. and Pearse, D.B. (2002) Treatment of Right Heart Thromboemboli. Chest, 121, 806-814. http://dx.doi.org/10.1378/chest.121.3.806

[13] Pierre-Justin, G. and Pierard, L.A. (2005) Management of Mobile Right Heart Thrombi: A Prospective Series. International Journal of Cardiology, 99, 381-388. http://dx.doi.org/10.1016/j.ijcard.2003.10.071

[14] Torbicki, A., et al. (2003) Right Heart Thrombi in Pulmonary Embolism: Results from the International Cooperative Pulmonary Embolism Registry. Journal of the American College of Cardiology, 41, 2245-2251. http://dx.doi.org/10.1016/S0735-1097(03)00479-0

[15] Ruel, M., et al. (2001) Resection of Right Atrial Tumor Thrombi without Circulatory Arrest. The Annals of Thoracic Surgery, 71, 733-734. http://dx.doi.org/10.1016/S0003-4975(00)02317-1

[16] Cale, R., et al. (2008) Giant Left Atrial Mass: Thrombus Mimicking Myxoma. Revista Portuguesa de Cardiologia, 27, 1191-1194.

\section{Abbreviations and Acronyms}

TTE: Transthoracic Echocardiography

TEE: Transesophageal Echocardiography

DVT: Deep Venous Thrombosis

TCD: Transcranial Doppler Sonography 